\title{
Enhanced MALDI Ionization Efficiency \\ at the Metal-Matrix Interface: Practical \\ and Mechanistic Consequences \\ of Sample Thickness and Preparation Method
}

\author{
Gregor McCombie and Richard Knochenmuss \\ Novartis Institutes of Biomedical Research, Basel, Switzerland
}

\begin{abstract}
Electrosprayed spots of varying thickness were evaluated for use as reproducible, homogenous, high efficiency MALDI samples. Thin samples on stainless steel plates were found to give exceptionally strong signals, as did the last layers of thick samples, when ablated down to the steel substrate. A small enhancement was also observed for thin samples on a gold substrate, and with a few-nanometer gold coating on top of a thick sample. Ion yields and intensity ratios can be understood in the context of the previously described quantitative MALDI model including the matrix-metal interfacial ionization potential reduction effect (Knochenmuss, R.; Anal. Chem. 2004, 76, 3179-3184). The absolute and relative stabilities of ion signals were found to be at least a factor of two better for the thin electrosprayed spots, compared to spots prepared by dried droplet methods. (J Am Soc Mass Spectrom 2006, 17, 737-745) () 2006 American Society for Mass Spectrometry
\end{abstract}

$\Lambda$ lthough matrix assisted laser desorption/ionization (MALDI) mass spectrometry (MS) has been highly successful and widely investigated since its introduction in 1987 [1], both the underlying ionization mechanisms and sample preparation methods remain objects of active study. These are coupled topics since mechanistic understanding can allow rational, rather than undirected, method development. Here, we make use of a recently developed quantitative model for UV-MALDI based on a two-step ionization process $[2,3]$. Primary laser-created matrix ions and analyte neutrals undergo ion-molecule reactions in the expanding ablation plume to yield secondary analyte ions. The observed mass spectrum is determined by the thermodynamics of these reactions. Because matrix and analyte are explicitly coupled, the model proved particularly suitable for understanding of practically relevant phenomena such as ion suppression effects [4-6], which have been shown to be very common phenomena [7].

A variant of this model has recently been developed for thin samples on metal substrates [8]. This was motivated by measurements of electron emission yields from MALDI samples on metallic versus insulating substrates [9], and suggestions that such electrons might neutralize (positive) analyte ions during the desorption process [10]. The model gave excellent agreement with the electron yield data, but this was not consistent with claims of

Published online March 20, 2006

Address reprint requests to Dr. R. Knochenmuss, Novartis Institutes for Biomedical Research, WSJ-503.11.04, Novartis Pharma AG, Postfach 4002 Basel, Switzerland.E-mail: Richard.Knochenmuss@novartis.com higher analyte ion yields for samples on nonconductive versus conductive substrates [9]. The major difference between thick and thin samples is the interaction of matrix molecules with the substrate, which can lower the ionization potential of the combined system if the substrate is metal. It was, therefore, theoretically expected that thin samples on metal would yield higher, not lower, signals. The present study was undertaken to investigate this discrepancy and restore consistency to sample preparation strategy.

The method selected here to prepare homogenous samples of variable thickness is electrospray (ES) deposition of analyte/matrix solution [11, 12]. Electrospray produces more uniform, fine-grained sample spots than the more convenient and widespread dried droplet technique, which tends to form large matrix crystals in random locations. By varying the electrospray time, it is also possible to make samples as thin as a few hundred nanometers, but a few $\mathrm{mm}$ in diameter.

Our investigation confirms the theoretical expectations. Thin samples on metal substrates are more easily ionized than thicker ones, and this leads reproducibly to significantly higher, not lower, analyte and matrix ion yields. The enhancement depends on the metal used, in a manner consistent with theory. We find no evidence for photoelectron neutralization of positive ions.

\section{Experimental}

The home-built ES deposition system used a CTC-PAL syringe autosampler (Zwingen, Switzerland) to dispense the analyte/matrix solution. After aspiration of 


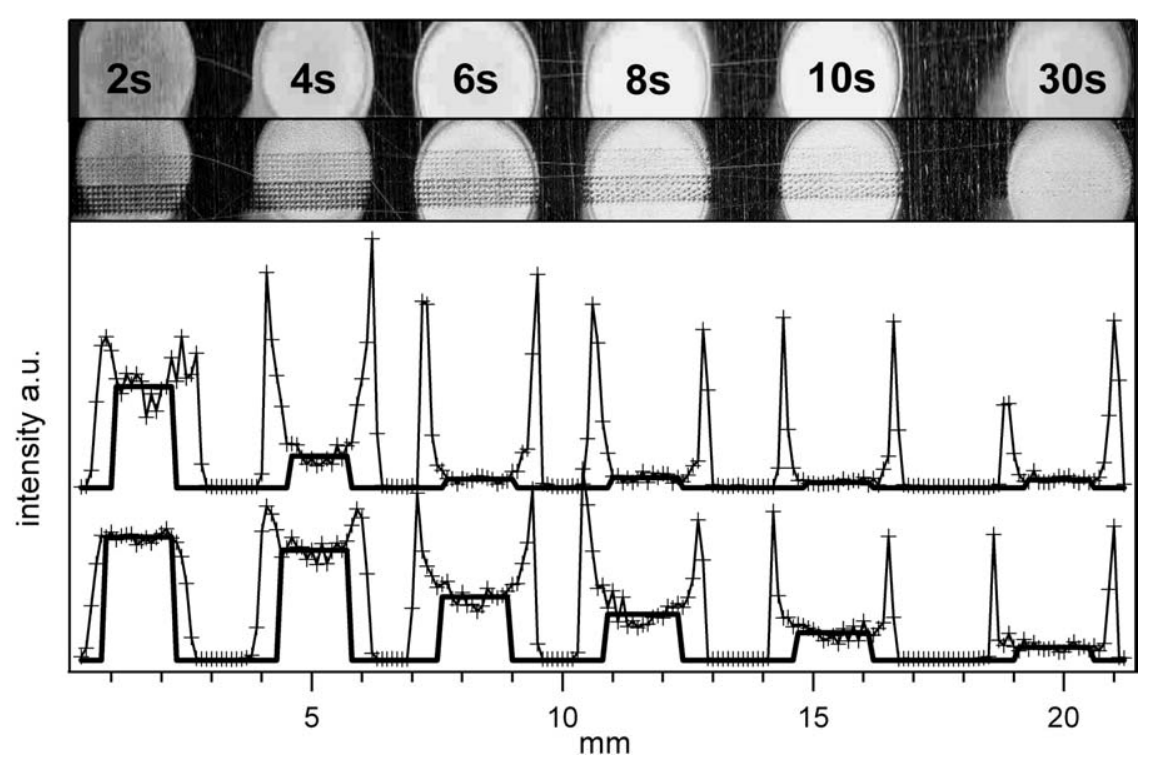

Figure 1. Electrosprayed MALDI spots of reserpine and Substance P in DHB matrix; ( $325 \mathrm{mM}$ DHB, $150 \mu \mathrm{M}$ Substance $\mathrm{P}, 25 \mu \mathrm{M}$ reserpine in methanol $/ \mathrm{CHCl}_{3} / \mathrm{H}_{2} \mathrm{O}$ 50:48:2). Spray times are indicated on the top row, which is an optical image of the spots before MS acquisition. The second image shows the sample after the MALDI measurement. Ten rows of laser ablation craters are visible, five each with 5 and 50 shots per crater. The distance between laser craters is $0.1 \mathrm{~mm}$. The upper traces in the lower panel show the Substance P signals summed over the five 5-shot columns. The corresponding data for the 50-shot columns are below. The traces with symbols are the measured values. In the regions where it is not set to zero, the solid line is the average value of the most uniform central region of each spot.

$10 \mu \mathrm{l}$ sample solution, $8.5 \mu \mathrm{l}$ were ejected rapidly onto an unused part of the sample plate to flush the tubing and spray needle. This spray was pneumatically assisted to prevent the formation of droplets that could leave residue on the needle. The MALDI spot was sprayed at a rate of $11 \mathrm{nl} / \mathrm{s}$, with no pneumatic assistance. The needle was positioned $6 \mathrm{~mm}$ above the target plate and a potential of $+5 \mathrm{kV}$ was applied. The target plate was moved manually between depositions.

All mass spectra were measured on a Voyager-DE STR MALDI TOF instrument, (Applied Biosystems, Framingham, MA). Reflectron mode and an acceleration voltage of $20 \mathrm{kV}$ were used. The acceleration pulse delay was $175 \mathrm{~ns}$. The $355 \mathrm{~nm}$ laser intensity was around 100 units over the apparent threshold value except for Figure 2, where it was about 100 units higher still. The fluences for all experiments were within the normal range for typical MALDI experiments. The digitizer bin size was set to 2 ns. Single spectra were exported to and analyzed using IGOR PRO (WaveMetrics, Lake Oswego, OR).

The MALDI instrument has been adapted for fast MS imaging [13]. For every image point, 5-50 shots (mass spectra) were averaged. The raster step size was 100 $\mu \mathrm{m}$. The images were directly analyzed using IGOR PRO (Wavemetrics).

The Substance $\mathrm{P} /$ reserpine sample solution was made up of $325 \mathrm{mM}$ DHB, $150 \mu \mathrm{M}$ Substance $\mathrm{P}, 25 \mu \mathrm{M}$ reserpine in methanol $/ \mathrm{CHCl}_{3} / \mathrm{H}_{2} \mathrm{O}$ 50:48:2. The lipid extract of mouse serum was performed using the method of Schiller et al. [14], modified from Bligh and
Dyer [15]. Twenty microliters of serum were vortexed with $75 \mu \mathrm{l}$ methanol $/ \mathrm{CHCl}_{3}$ 2:1 for $1 \mathrm{~min}$. After addition of $25 \mu \mathrm{lCHCl}_{3}$ the sample was vortexed again for $1 \mathrm{~min}$. Then $25 \mu \mathrm{l}$ of $40 \mathrm{mM}$ acetic acid in water was added and the sample was vortexed for an additional 1 min. After centrifugation the organic layer was used to prepare the MALDI sample.

The matrix suppression effect (MSE) score was used in some cases to evaluate the many spectra acquired. It is defined as the summed analyte signals over the total ion current from both matrix and analyte [7]:

$$
M S E_{\text {score }}=\frac{\sum A}{\sum A+\sum M}
$$

Where $\mathrm{M}$ are the intensities of predefined matrix signals and A those of analyte. The MSE score calculation was programmed in the intrinsic language of IGOR PRO (WaveMetrics). The expected masses of analyte and matrix were entered before analysis. These could include the protonated/deprotonated molecule, metal ion adducts, and all isotopes. Peaks were only detected if their intensity exceeded $1 / 30$ of the base peak. A MSE score of zero indicates that no analyte was found; a score of one indicates that no matrix signals were present, and matrix suppression was observed.

\section{Results}

Figure 1 shows six spots of the same solution, electrosprayed onto stainless steel for varying times to control 


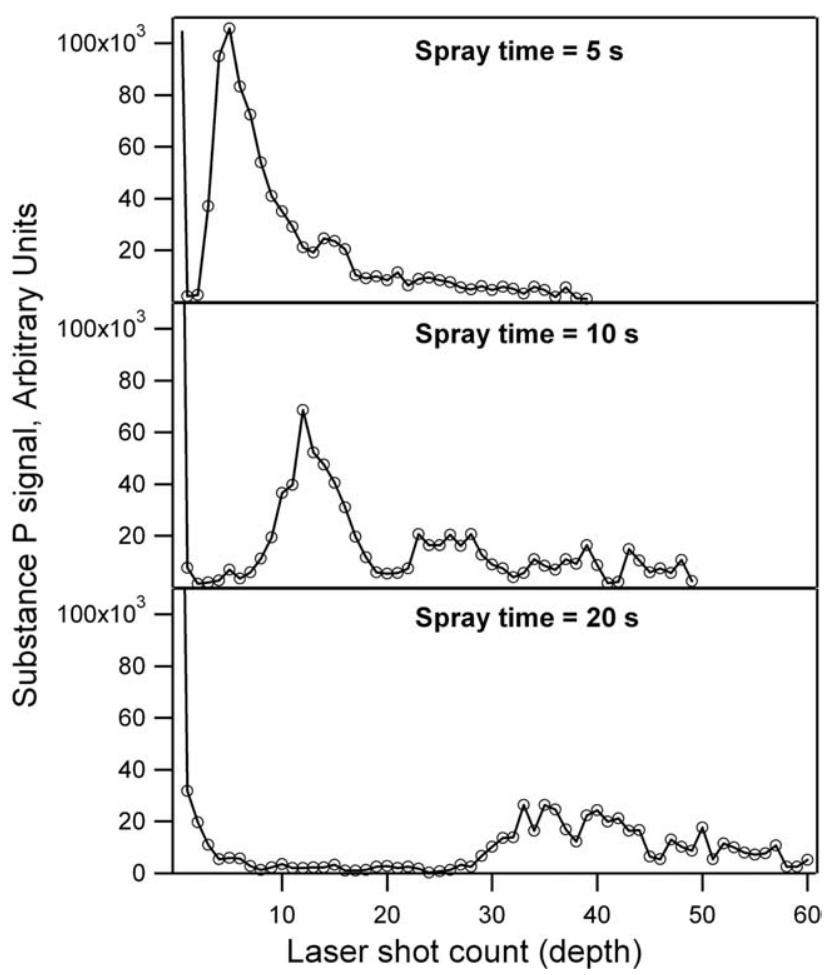

Figure 2. Substance P MALDI signals for sequential single laser shots on electrosprayed samples of varying thickness. The sample was $180 \mu \mathrm{M}$ Substance $\mathrm{P}$ and $50 \mathrm{mg} / \mathrm{ml}$ DHB (325 mM) in methanol/chloroform/water 50:47:3. After a high intensity for the first shot the signal drops until the laser reaches the metal plate, where the signal increases again.

the thickness of the sample. In spite of their polycrystalline nature, the thinnest spots were sufficiently flat and transparent so that circular colored interference fringes could be observed. From the radial fringe spacing it could be inferred that the samples have a slightly domed form with a relatively steep outer edge. From the spray flow rate and the diameter of the spots, approximate thicknesses could be estimated, and correspond to a mean deposition rate of about $100 \mathrm{~nm} / \mathrm{s}$.

A very consistent feature of Figure 1 is that thinner sample regions give distinctly higher signals. This is apparent not only from the mean signal of the central regions versus spray time/thickness, but also from the sharp increase in signal at the thinner edges of the spots. Closer inspection shows that the highest signals are observed for those ablation sites where the laser has penetrated to the stainless steel substrate. Compare, for instance, the $6 \mathrm{~s}$ spots. Fifty laser shots were sufficient to ablate through the sample (bottom), while five were not (top). The total signal is about nine times higher for the fully ablated rows.

The data of Figure 1 strongly suggest that the sample layers nearest the metal give a large fraction of the integrated ion signal. If so, a laser drilling experiment should show a significant increase in yield when the ablation crater reaches the substrate. This is demonstrated in Figure 2 for spots of different thickness.
Substance P exhibits a strong "first shot effect" [16] in DHB matrix, suggesting some degree of segregation on the surface. Alternatively, the first shot may ablate the smallest crystallites with disproportionate efficiency and yield [17]. Following the first shot, analyte signal drops rapidly to a low value, where it remains until shortly before the substrate is reached. Then a large peak appears, as expected from Figure 1. The peak has a significant tail since the ablation crater is conical rather than flat, so after initial contact with the surface, the edges of the crater also slowly ablate down to the metal [18].

In addition to the overall signal level, relative intensities of matrix and analytes are also important for spectral quality, as is the reproducibility of the results over a sample spot and between spots. MALDI imaging $[13,19]$ was therefore used to examine thick and thin electrosprayed spots, as seen in Figure 3. The molar matrix to analyte ratio of 150 is known to give a substantial matrix suppression effect (MSE) with a laser fluence near threshold, for thick dried droplet samples [7]. This is also the case here for the thick electrosprayed spot. In contrast, the MSE scores for the thin spot are low across the entire surface. (The ring with a high MSE score is an artifact. The edge is so thin and signal is so weak that the DHB signals were lost in the noise.) It is

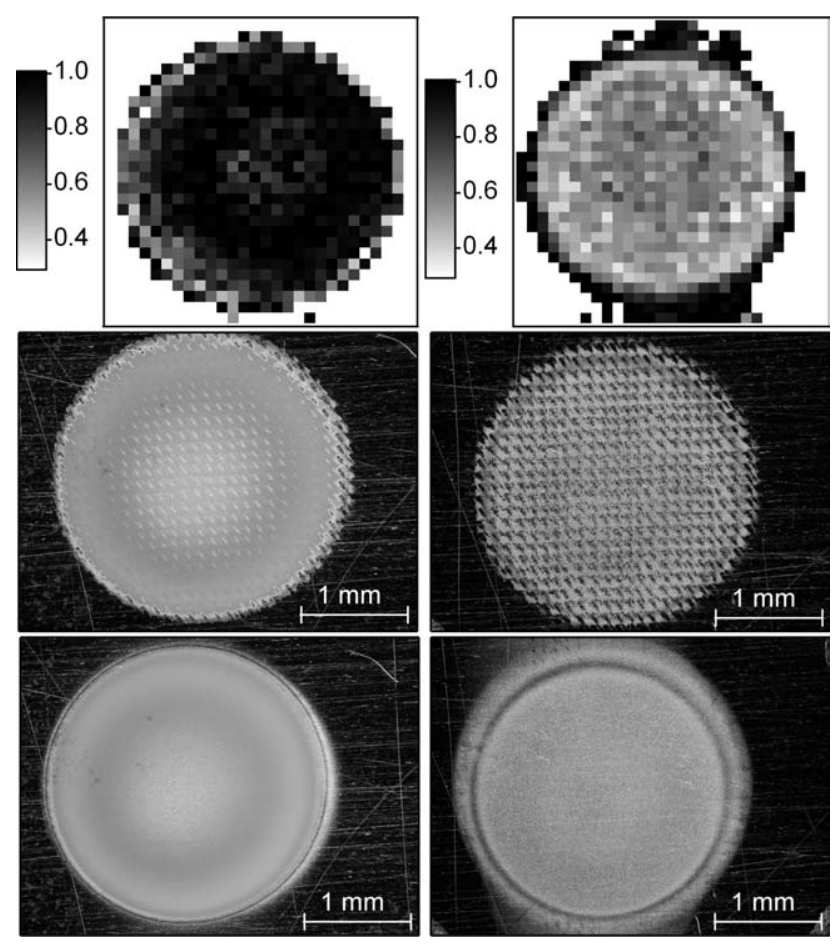

Figure 3. Optical and MALDI images of thick (left) and thin (right) electrosprayed samples. The solution of $280 \mathrm{mM}$ DHB, 1.9 $\mathrm{mM}$ reserpine in methanol/ $\mathrm{CHCl}_{3}$ 1:1 was sprayed for $40 \mathrm{~s}$ and $5 \mathrm{~s}$, respectively. Fifty laser shots were summed for each pixel. The bottom micrographs show the spots before MALDI imaging, the middle row after imaging. The top row is a matrix suppression effect (MSE) score quantifying the relative matrix and analyte signal intensities [7]. The MALDI instrument settings were identical for both images. 


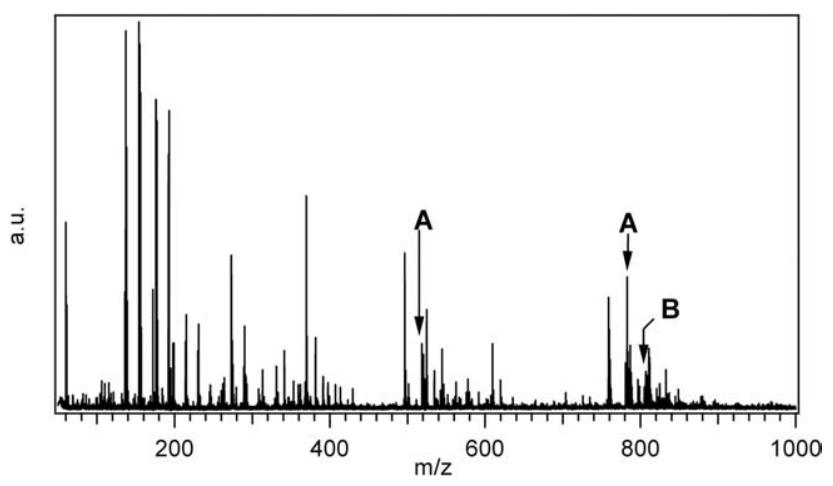

Figure 4. MALDI mass spectrum (positive ion mode) of mouse serum lipid extract in DHB matrix. The sample was prepared by electrospray as described in the text. The marked signals were used in the analysis of Figure 5.

important to note that the image acquisition parameters such as the laser intensity were the same for both images. The effect of a thin sample is, therefore, not only a gain in intensity but also a reduction in MSE. This appears to be analogous to the reduction of MSE by increasing the laser fluence [7]. In both cases, larger amounts of primary matrix ions are created, more than can be consumed by reaction with analyte neutrals.

Relative analyte signals are also important for MALDI applications and were investigated using serum lipid extract, as shown in Figure 4. Two analytes (marked A) were selected for comparing peak ratio variability in two identically prepared spots. As seen in the lower trace of Figure 5, the absolute signal levels vary somewhat across each spot, but the middle trace

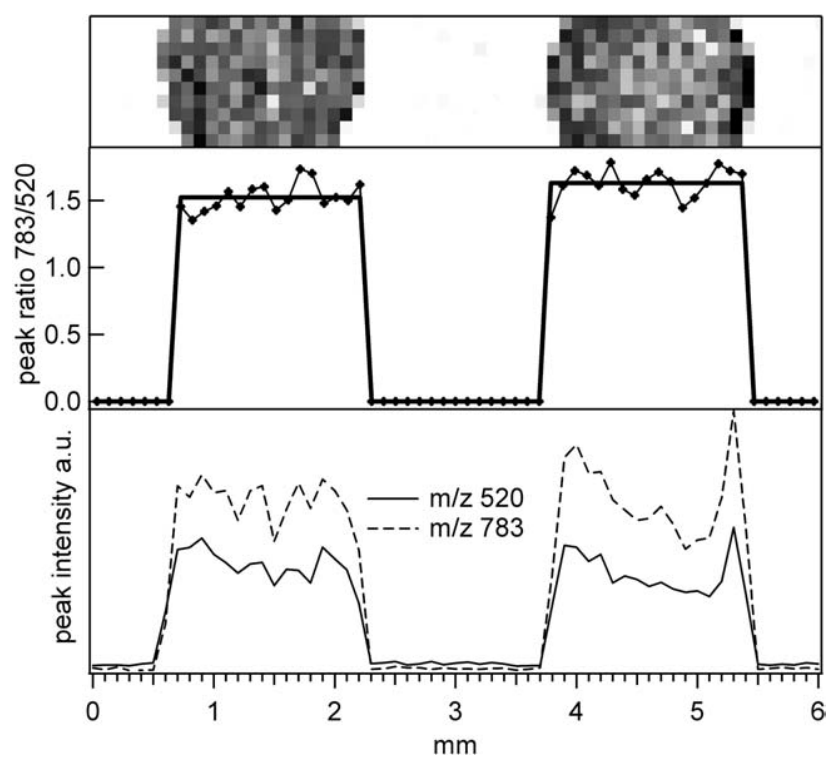

Figure 5. Top: MALDI image of 2 electrosprayed spots of serum lipid extract in DHB. The contrast is based on the intensity of peak B of Figure 4 . The spray time was $12 \mathrm{~s}$. Bottom: summed intensities for the two peaks marked A in Figure 4 over all pixels of each column. Middle: Peak ratios of the two signals in the bottom row. The symbols are the raw values and the solid line the average per spot.

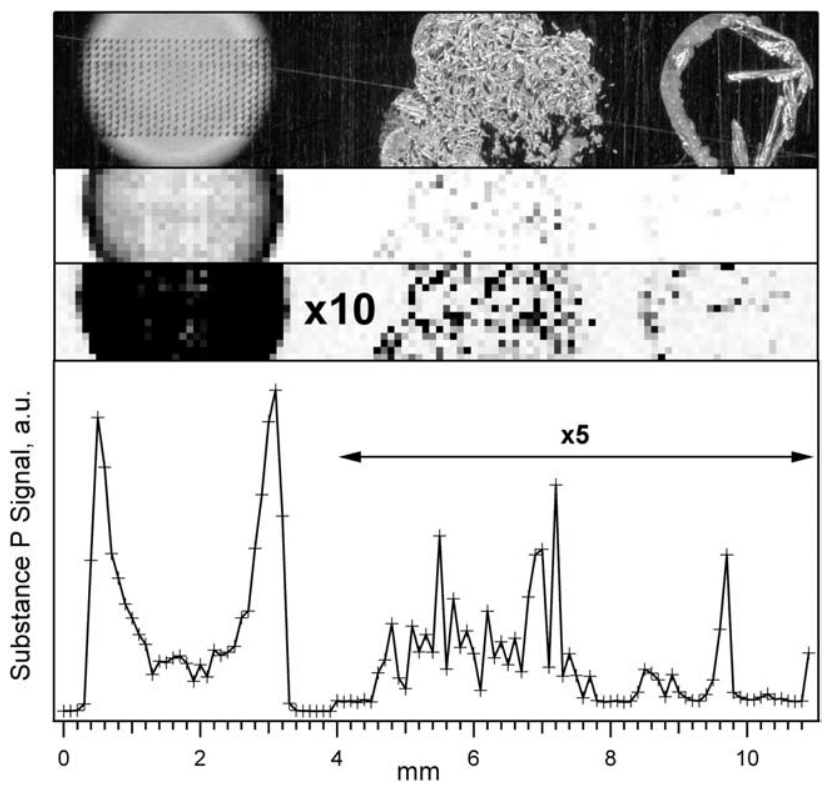

Figure 6. Comparison of three MALDI sample preparation methods. The same solution was used for the left and middle spots: Substance P $180 \mu \mathrm{M}$, with matrix DHB $50 \mathrm{mg} / \mathrm{ml}(325 \mathrm{mM})$ in chloroform/methanol/water 48.5:50:1.5. The left spot was electrosprayed for $8 \mathrm{~s}$, the middle was made by fast drying of a $0.75 \mu \mathrm{l}$ droplet, and the right spot made by slow drying of a droplet in methanol/water 1:1 with the same analyte and matrix concentrations. The MALDI images ( 2 middle rows) show the intensities of the Substance P signal. Twenty laser shots were summed for each pixel. The lower trace shows the summed intensities for this analyte over all image columns.

demonstrates that the laterally averaged peak ratios are very reproducible. The MALDI images show some mild pixel-to-pixel variation in the peak ratio, but this is spatially random, not systematic.

This is a very high degree of homogeneity and reproducibility for MALDI samples $[12,20]$. The customary dried droplet method is seldom so effective, especially for matrices such as DHB that do not leave evenly distributed crystals upon drying. The relatively slow crystallization process can lead to preferential enrichment of some analytes at the edge and others in the center of the spot [7]. Figure 6 compares electrospray, fast dried drop, and slow dried drop samples, again for a sample of Substance P. The two MALDI image rows demonstrate that the thin electrosprayed sample yields considerably higher signal of Substance P across the spot and is far more homogenous as well. The lower trace is a sum over all pixels at a given $x$-value, showing in a more quantitative fashion the signal strength advantage of the thin electrosprayed spot. Considering the center part of the sprayed spot and only those pixels from the other two in which the $\mathrm{P}$ signal is greater than the noise threshold, the average signals versus spray $=100$ (with standard deviation) are: 100 (37), 48 (69), and 35 (91), from left to right. The stability advantage of the sprayed spot is clear, even neglecting pixels of the dried drops that give no signal. 


\section{Discussion}

\section{Thin Sample on Metal}

MALDI ionization yield at the matrix-metal interface has already been considered in another context, that of electron emission from MALDI samples [8]. Electronic states of matrix molecules interact with the metal conduction band to lower the ionization potential of the matrix, as illustrated in Figure 7 for DHB matrix. While ionization in bulk matrix is energetically a three-photon event, [2] for the surface layer it can become twophoton. Simultaneous two- or three-photon excitation is not required, energy storage in the first excited-state and exciton hopping allow sequential multiphoton processes to be important at rather low laser intensities [21].

The reduction in the order of the matrix ionization process from three to two makes it much more efficient at a given laser intensity, as will be discussed quantitatively below. If matrix ion yield is increased, ionmolecule reactions lead to increased analyte ion yield as well, as has been shown and modeled in detail elsewhere:[2-6, 22].

$$
\mathrm{M}^{+}+\mathrm{A} \rightarrow \mathrm{M}+\mathrm{A}^{+}
$$

The simplest condition for two-photon ionization of the surface layer is that LUMO-derived orbitals fall below the Fermi level and become populated. As is clear from Figure 7, this is always the case for DHB on stainless steel, since the LUMO itself is $0.1 \mathrm{eV}$ below the Fermi level. Every DHB molecule that is within tunneling range of the surface will exhibit a low ionization potential. This picture is consistent with the data shown above. DHB on stainless steel is found to uniformly exhibit a strong surface-related signal enhancement.

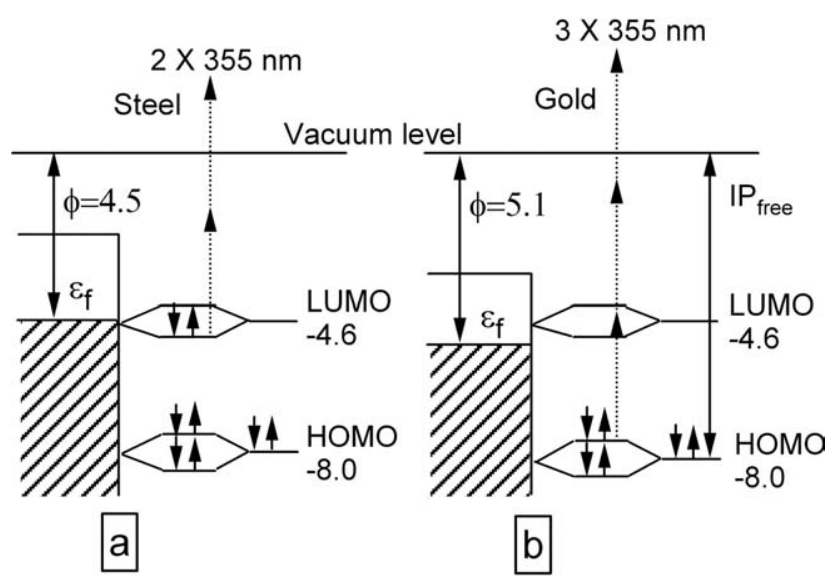

Figure 7. Schematic of molecular orbitals of DHB adsorbed on the surface of stainless steel (a) and gold (b). The vertical axis is to scale, with energy in $\mathrm{eV}$ versus the vacuum level. The metal conduction band is on the left of each panel, free DHB on the right. The free DHB ionization potential and LUMO energy are from reference [32]. Adsorbed DHB on the metal surface is in the center, the state splittings due to interaction with the metal are approximate.

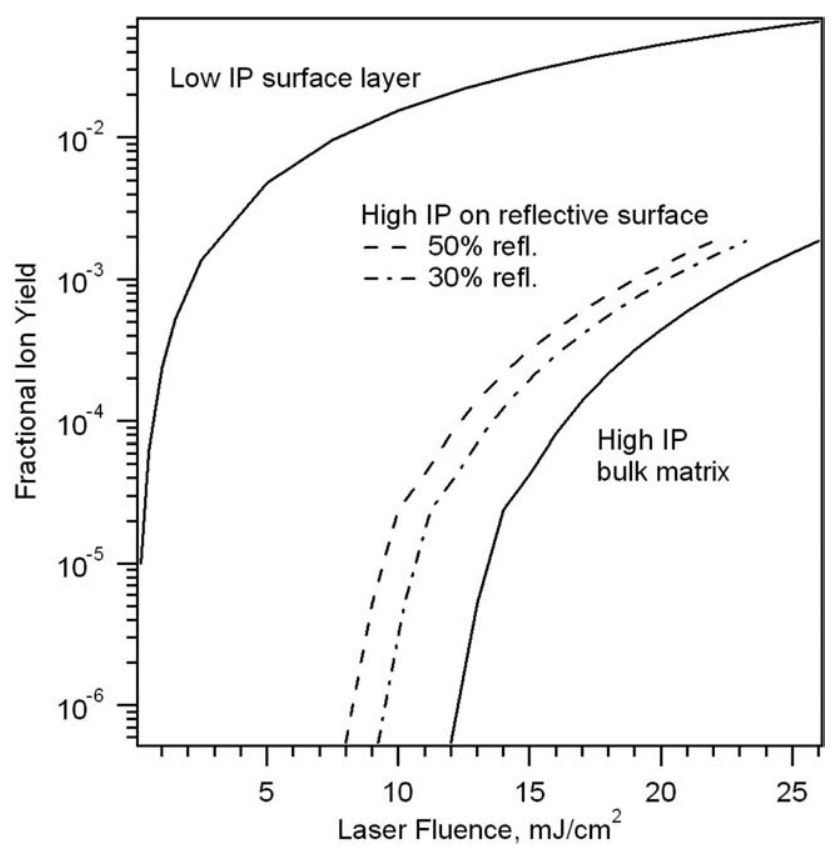

Figure 8. MALDI primary ion yields from the bulk matrix surface (lower solid trace), and from a thin-layer of matrix on a metal substrate (upper solid trace). The calculations were performed with the models of references $[3,8]$. The laser wavelength was $355 \mathrm{~nm}$ and the matrix was DHB. Ionization is energetically three-photon in the bulk and two-photon for the surface layer. The dashed lines account for effective fluence increase due to reflection of the substrate surface (30\% represents gold and 50\% stainless steel). See the text for further details.

Since the relevant properties are known for DHB, the surface-enhanced MALDI ion yield can be modeled [8], as shown in Figure 8. This is predicted to be about two orders of magnitude higher than for bulk matrix, at typical MALDI laser fluences toward the right end of Figure 8 . The experimental single shot increase of the surface layer signal versus higher layers can reach two orders of magnitude as seen in the top panel of Figure 2. When multiple shots are summed, as in the images shown above, net enhancements of about one order of magnitude are typical, since not only the lowest layer is sampled.

One test of this model is to take a different metal as sample substrate. Gold is widely used due to its relative chemical inertness. Figure 9 shows a MALDI image of samples of varying thicknesses, similar to Figure 1. Half of the substrate was native stainless steel, and half sputter coated with gold $(\sim 35 \mathrm{~nm})$. The familiar thinlayer signal increase on steel is again apparent, but much less pronounced on gold. Nevertheless, the gold half of each image does show an enhanced ring on the outer, thinnest part of the sample, so the phenomenon occurs on this substrate as well.

A hypothesis for the smaller enhancement on gold is shown in Figure 7. The Fermi level of gold lies $0.6 \mathrm{eV}$ lower than that of steel [23], so any ionization potential reduction depends on the degree of DHB LUMO splitting due to surface interactions. If it is small, the surface 


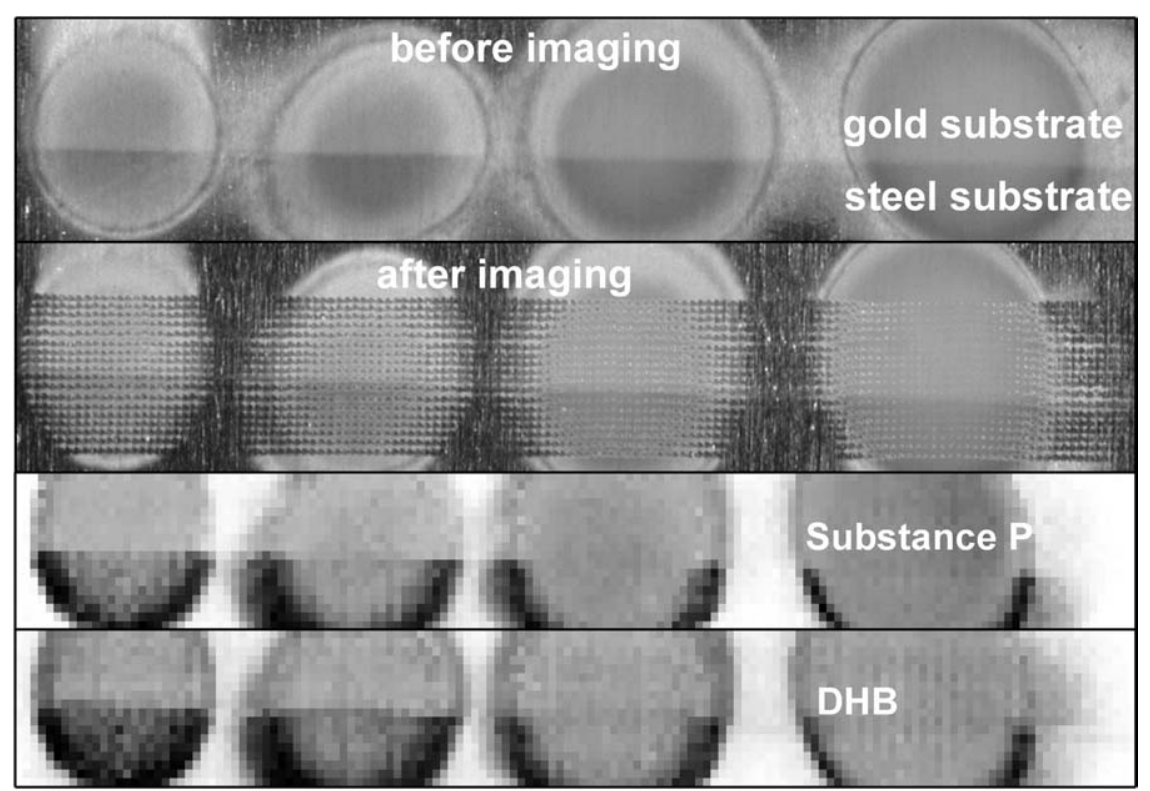

Figure 9. Electrosprayed samples of varying thickness similar to Figure 1. The top two rows are micrographs of the samples before and after MALDI image analysis. The stainless steel substrate was covered with $35 \mathrm{~nm}$ gold on the top half before sample deposition. The bottom two rows are MALDI images based on the DHB and Substance P signal as labeled on the figure. The sample solution was the same as for Figure 6. The spectrum of each pixel was the sum of 50 laser shots.

states will not be populated, and IP reduction will be minimal. If it is near $\mathrm{kT}$ (including the surface heating by the laser) of the Fermi level, some adsorbed matrix molecules will exhibit enhanced ionization efficiency, but not all. This situation is consistent with the weak gold ionization enhancement. Apparently, only a fraction of the DHB adsorbed on gold is able to undergo efficient two-photon ionization.

\section{Metal Coating on Thick Sample}

The above data have demonstrated that the near-metal matrix layer yields high MALDI signal. This should be true not only for the matrix on a monolithic substrate, but matrix near any metallic structure with an appropriate Fermi level. It has been demonstrated recently that MALDI imaging can benefit from a few-nm surface coating of gold or other metal (Markus, Stoeckli; in preparation).

An example is shown in Figure 10, where half of a thick electrosprayed sample was overcoated with gold. The signal enhancement may be partially associated with decreased surface charging [24] but charging affects primarily the ion flight times and not the total ion signal. The coating process creates many microscopic regions of metal-matrix contact, all at the top of the sample where the laser intensity is highest. This is a situation that is advantageous for the interfacial IP reduction effect.

On close inspection, the gold overcoat region in Figure 10 appears to give a slightly greater average enhancement than the thin sample regions on top of gold in Figure 9. Comparing inner and edge regions of a thick spot in Figure 9, an underlayer enhancement factor of $1.3 \pm 0.3$ was calculated. For the gold coated halves of a spot in Figure 10 the corresponding factor is $1.6 \pm 0.8$. The difference is not presently statistically significant, but might warrant further investigation. The gold overlayer is probably not homogenous but rather forms small islands [25], which may be strongly heated by the laser (see below). It is then possible that it is acts as a thermal desorption substrate, similar to the cobalt particles used by Tanaka et al. in early biomolecule desorption/ionization experiments [26], or the graphite particles that have subsequently achieved broader application [27]. Other mechanisms such as charge scavenging and subsequent transfer to analytes can be postulated but cannot be addressed with the present data.

\section{Surface Reflection}

In addition to the surface state model proposed above, increased yield due to laser reflection at the metal surface was considered. The $1 /$ e penetration depth of $355 \mathrm{~nm}$ light in DHB is about $350 \mathrm{~nm}[21,28,29]$, so the last layers of matrix are relatively transparent. Reflection from the metal, therefore, increases the effective fluence for these layers. Figure 8 compares the IP reduction model with the ion yield omitting surface interaction, but with an increased effective laser intensity due to reflection from the surface. The intensity is increased by the reflectivity of the surface minus any absorption by the matrix. The reflectivity is $50 \%$ for 


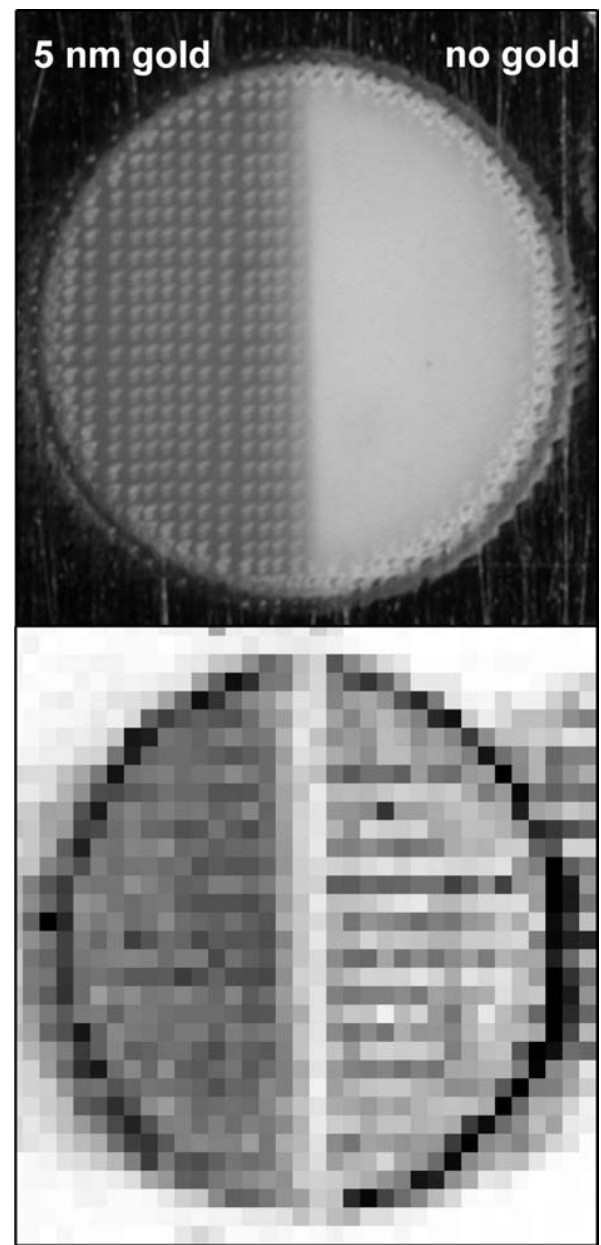

Figure 10. Optical image (upper) and DHB $\left(\mathrm{MH}^{+}\right)$MALDI image (lower) of an electrosprayed sample on stainless steel. The sample solution was the same as for Figure 6. After $30 \mathrm{~s}$ electrospray, $5 \mathrm{~nm}$ of gold was sputter deposited on top of the left half. Fifty laser shots were summed for each spectrum of the image.

stainless steel [30] and 35\% for gold (e.g., www.micd.com/curriculum/lightandcolor/mirrors.html) at 355 $\mathrm{nm}$, equivalent to a shift of the bulk matrix ionization efficiency curve to the left (dashed curves). For typical fluences, this gives modest yield increases of less than an order of magnitude. As shown above for the drilling experiment, this is insufficient to account for the effects observed.

\section{Surface Heating}

Consider first a thin metal layer comparable to the evaporated gold overcoat above. If the layer is of a thickness comparable to the skin depth, all energy deposited remains in the excited volume, and the temperature increase due to the laser pulse is:

$$
\Delta T=I_{0} \frac{\left(1-f_{r}\right)}{C_{p} \rho A \delta}
$$

Where $I_{o}$ is the incident laser pulse energy, $f_{r}$ is the fraction of reflected energy, $C_{p}$ the heat capacity, $\rho$ the density, A the irradiated area, and $\delta$ the skin depth (5 $\mathrm{nm}$ for gold, approximately $30 \mathrm{~nm}$ for stainless steel). The ratio $\Delta \mathrm{T}(\mathrm{Au}) / \Delta \mathrm{T}(\mathrm{SS})$ is independent of pulse energy and spot area, and has the value 12.7 , that is, a gold layer exhibits a much bigger temperature jump than a steel layer.

Thermal conductivity decreases the peak temperature of a thick metal layer during a nanosecond laser pulse, since thermal diffusion over short distances is fast. The general diffusion equation can be reduced to one-dimensional form for the present problem, since the skin depth is very small compared to the laser spot diameter:

$$
\frac{d T}{d t}=a \frac{d^{2} T}{d x^{2}}
$$

Where $a=\lambda / \rho C_{p}$ and $\lambda$ is the thermal conductivity (317 W/(m-K) for gold and 13-16 for SS). For a very short laser pulse, an exponential temperature distribution is created in the metal with a $1 / \mathrm{e}$ depth $=\delta$. For gold, $\mathrm{dT} / \mathrm{dt}$ is then $2 \times 10^{12} \Delta \mathrm{T}(\mathrm{K} / \mathrm{s})$, where $\Delta \mathrm{T}$ is the temperature difference between the surface and depth $\delta$. This diffusion rate shows that a $35 \mathrm{~nm}$ gold layer on steel, as used in the above experiments, is not the limiting factor in thermal relaxation from the irradiated surface. The gold layer becomes nearly uniformly heated during a few-ns $355 \mathrm{~nm}$ laser pulse, and further thermal energy transport into the bulk is limited by the conductivity of the underlying stainless steel. The peak temperature ratio for the gold and SS surfaces used in our experiments is therefore as calculated above, but with the gold skin depth replaced by the layer thickness. The ratio $\Delta \mathrm{T}(\mathrm{Au}) /$ $\Delta \mathrm{T}$ (SS) then has the value 2 . The gold surface becomes at least as hot as the steel surface.

Finally, the theoretical considerations above were confirmed by 1-dimensional finite element simulations. Stainless steel and $35 \mathrm{~nm}$ gold on stainless were simulated under irradiation by a $6 \mathrm{~ns}$ full width, $355 \mathrm{~nm}, 40$ $\mathrm{mJ} / \mathrm{cm}^{2}$ temporally Gaussian laser pulse. As expected, the peak temperature of the thin gold layer was higher than that of the uncoated steel: 665 versus $610 \mathrm{~K}$ (initial temperature $=298 \mathrm{~K}$ ).

The above considerations show that a thermal mechanism is incompatible with the observed differences in ionization behavior on gold versus steel, or with a gold overcoat. The latter case is particularly notable; the substantially higher temperature that should be reached in the thin overcoat versus the bulk metal surfaces ought to have a dramatic effect on ionization efficiency in a thermal model. Instead, a statistically insignificant difference was observed.

\section{Sample Morphology versus Thickness}

While electrospray generally gives quite uniform samples, vertically stratified morphology variations 
may be possible. Since morphology can play a role in MALDI efficiency $[17,31]$, this factor was also considered. The hypothesis is that near-surface crystals might be more MALDI-active than bulk or top layer crystals because of varying formation processes during electrospray.

Strong evidence against this hypothesis is found in Figure 10. Matrix crystal morphology of the thick sample with the gold overcoat cannot be correlated with surface effects during electrospray, yet metalinduced enhancement is clearly observed. In addition, optical micrographs of our samples were taken after preparation and after MALDI measurement (i.e., several hours in vacuum). Both unpolarized and polarized light were used. Polarization increases contrast between crystals, which aids in evaluation of sizes and distributions.

While more small crystals were present in fresh samples compared to those after several hours in vacuum, no difference was observed at any time in crystal sizes or shapes between thin or thick samples. This qualitative conclusion was supported by analysis of the micrographs by 2-D Fourier transforms and particlefinding algorithms.

\section{Conclusions}

This work has explored practical and mechanistic aspects of electrospray MALDI sample preparation techniques on metal substrates. Using DHB as matrix, substantially increased ionization efficiency for thin samples on stainless steel was found, compared to thick samples. Ion suppression effects are also reduced and signal ratios are more stable. Comparison with dried droplet preparations demonstrated a factor of 10-100 better signal for the thin electrospray method, and much better homogeneity and reproducibility.

The thin sample enhancement effect is also observed on gold substrates or for thick samples with a gold overcoat, but is much weaker. The observations can be rationalized by a model based on a matrix-metal interfacial IP reduction effect $[2,3,8]$. DHB matrix ionization requires the energy of three $355 \mathrm{~nm}$ photons in the bulk, but only two at the steel surface. This increases DHB ionization rates at the surface, and analyte yield is subsequently increased by ion-molecule reactions with matrix ions. Enhancement is dependent on population of matrix-metal surface states, leading to the different behavior of gold and steel substrates. It should also vary with matrix choice. Yields of thick versus thin samples on steel are well described by a quantitative model derived from that used for the bulk matrix $[2,3$, 8].

Alternative mechanisms for the surface-enhanced yield, such as laser reflection at the surface, metaldependent surface heating, and vertically stratified ma- trix crystal morphology were evaluated and found to be incompatible with the data. Since ionization enhancement is observed in positive polarity, these results raise substantial questions regarding reports of reduced signal via neutralization by photoelectrons from the metal.

\section{References}

1. Karas, M.; Bachmann, D.; Bähr, U.; Hillenkamp, F. Matrix-Assisted Ultraviolet Laser Desorption of Nonvolatile Compounds. Int. J. Mass Spectrom. Ion Processes 1987, 78, 53-68.

2. Knochenmuss, R. A Quantitative Model of Ultraviolet Matrix-Assisted Laser Desorption and Ionization. J. Mass Spectrom. 2002, 37, 867-877.

3. Knochenmuss, R. A Quantitative Model of UV-MALDI Including Analyte Ion Generation. Anal. Chem. 2003, 75, 2199.

4. Knochenmuss, R.; Karbach, V.; Wiesli, U.; Breuker, K.; Zenobi, R. The Matrix Suppression Effect in Matrix-Assisted Laser Desorption/Ionization: Application to Negative Ions and Further Characteristics. Rapid Commun. Mass Spectrom. 1998, 12, 529-534.

5. Knochenmuss, R.; Dubois, F.; Dale, M. J.; Zenobi, R. The Matrix Suppression Effect and Ionization Mechanisms in Matrix-Assisted Laser Desorption/Ionization. Rapid Commun. Mass Spectrom. 1996, 10, 871877

6. Knochenmuss, R.; Stortelder, A.; Breuker, K.; Zenobi, R. Secondary Ion-Molecule Reactions in MALDI. J. Mass Spectrom. 2000, 35, 12371245.

7. McCombie, G.; Knochenmuss, R. Small-Molecule MADI Using the Matrix Suppression Effect to Reduce or Eliminate Matrix Background Interferences. Anal. Chem. 2004, 76, 4990-4997.

8. Knochenmuss, R. Photoionization Pathways and Free Electrons in UV-MALDI. Anal. Chem. 2004, 76, 3179-3184.

9. Frankevich, V. E.; Zhang, J.; Friess, S. D.; Dashtiev, M.; Zenobi, R. Role of Electrons in Laser Desorption/Ionization Mass Spectrometry. Anal. Chem. 2003, 75, 6063-6067.

10. Karas, M.; Glückmann, M.; Schäfer, J. Ionization in MALDI: Singly Charged Molecular Ions are the Lucky Survivors. J. Mass Spectrom. 2000, 35, 1-12.

11. Hensel, R. R.; King, R. C.; Owens, K. G. Electrospray Sample Preparation for Improved Quantitation in Matrix-Assisted Laser Desorption/ Ionization Time-of-flight Mass Spectrometry. Rapid Commun. Mass Spectrom. 1997, 11, 1785-1793.

12. Axelsson, J.; Hoberg, A.-M.; Waterson, C.; Myatt, P.; Shield, G. L.; Verney, J.; Haddleton, D. M.; Derrick, P. J. Improved Reproducibility and Increased Signal Intensity in Matrix-assisted Laser Desorption/ Ionization as a Result of Electrospray Sample Preparation. Rapid Commun. Mass Spectrom. 1997, 11, 209-213.

13. Stoeckli, M.; Staab, D.; Staufenbiel, M.; Wiederhold, K. H.; Signor, L. Molecular Imaging of Amyloid $\beta$ Peptides in Mouse Brain Sections Using Mass Spectrometry. Anal. Biochem 2002, 311, 33-39.

14. Schiller, J.; Müller, K.; Süss, R.; Arnhold, J.; Gey, C.; Herrmann, A.; Lessig, J.; Arnold, K.; Müller, P. Analysis of the Lipid Composition of Bull Spermatozoa by MALDI-TOF Mass Spectrometry-A Cautionary Note. Chem. Phys. Lipids 2003, 126, 85-94.

15. Bligh, E. G.; Dyer, W. J. A Rapid Method of Total Lipid Extraction and Purification. Can. J. Biochem. Phys. 1959, 37, 911-917.

16. Cohen, L. H.; Strupat, K.; Hillenkamp, F. Analysis of Quaternary Protein Ensembles by Matrix-Assisted Laser Desorption/Ionization Mass Spectrometry. J. Am. Soc. Mass Spectrom 1997, 8, 1046-1052.

17. Sadeghi, M.; Vertes, A. Crystallite Size Dependence of Volatilization in Matrix-Assisted Laser Desorption Ionization. Appl. Surf. Sci. 1998, 127/129, 226-234.

18. Fournier, I.; Marinach, C.; Tabet, J.-C.; Bolbach, G. Irradiation Effect in MALDI, Ablation, Ion Production, and Surface Modifications. II. 2,5Dihydroxybenzoic Acid Monocrystals. J. Am. Soc. Mass Spectrom. 2003, 14, 893-899.

19. Garden, R. W.; Sweedler, J. V. Heterogeneity within MALDI Samples as Revealed by Mass Spectrometric Imaging. Anal. Chem. 2000, 72, 30-36.

20. Gusev, A. I.; Wilkinson, W. R.; Proctor, A.; Hercules, D. M. Improvement of Signal Reproducibility and Matrix/Comatrix Effects in MALDI Analysis. Anal. Chem. 1995, 67, 1034-1041.

21. Setz, P. D.; Knochenmuss, R. Exciton Mobility and Trapping in a MALDI Matrix. J. Phys. Chem. 2005, 109, 4030-4037.

22. Knochenmuss, R.; Zenobi, R. MALDI Ionization: The Role of In-Plume Processes. Chem. Rev. 2003, 103, 441-452.

23. Hoffmann, R. Solids and Surfaces, a Chemist's View of Bonding in Extended Structures; VCH: Weinheim, 1988; p 142

24. Scherl, A.; Zimmermann-Ivol, C. G.; Di Dio, J.; Vaezzadeh, A. R.; Binz, P.-A.; Amez-Droz, M.; Cochard, R.; Sanchez, J.-C.; Glueckmann, M.; Hochstrasser, D. F. Gold Coating of Nonconductive Membranes Before Matrix-Assisted Laser Desorption/Ionization Tandem Mass Spectrometric Analysis Prevents Charging Effect. Rapid Commun. Mass Spectrom. 2005, 19, 605-610.

25. Delcorte, A.; Medard, N.; Bertrand, P. Organic Secondary Ion Mass Spectrometry: Sensitivity Enhancement by Gold Deposition. Anal. Chem. 2002, 74, 4955-4968. 
26. Tanaka, K.; Waki, H.; Ido, Y.; Akita, S.; Yoshida, Y.; Yoshida, T. Protein and Polymer Analyses of up to $\mathrm{m} / \mathrm{z} 100,000$ by Laser Ionization Time-of-Flight Mass Spectrometry. Rapid Commun. Mass Spectrom. 1988, 2, 151-153.

27. Sunner, J.; Dratz, E.; Chen, Y.-C. Graphite Surface-Assisted Laser Desorption/Ionization Time-of-Flight Mass Spectrometry of Peptides and Proteins from Liquid Solutions. Anal. Chem. 1995, 67, 4335-4342

28. Horneffer, V.; Dreisewerd, K.; Lüdemann, H.-C.; Hillenkamp, F.; Lage, M.; Strupat, K. Is the Incorporation of Analytes into Matrix Crystals a Prerequisite for Matrix-Assisted Laser Desorption/Ionization Mass Spectrometry? A Study of Five Positional Isomers of Dihydroxybenzoic Acid. Int. J. Mass Spectrom. 1999, 185/186/187, 859-870.
29. Allwood, D. A.; Dreyfus, R. W.; Perera, I. K.; Dyer, P. E. UV OpticalAbsorption of Matrices Used for Matrix-Assisted Laser Desorption/ Ionization. Rapid Commun. Mass Spectrom. 1996, 10, 1575-1578.

30. Karlsson, B. Ribbing, C. G. Optical Constants and Spectral Selectivity of Stainless Steel and Its Oxides. J. Appl. Phys. 1982, 53, 63406346.

31. Luxembourg, S. L.; McDonnell, L. A.; Duursma, M. C.; Xinghua, G.; Heeren, R. M. A. Effect of Local Matrix Crystal Variations in MatrixAssisted Ionization Techniques for Mass Spectrometry. Anal. Chem. 2003, 75, 2333-2341.

32. Karbach, V.; Knochenmuss, R. Do Single Matrix Molecules Generate Primary Ions in Ultraviolet Matrix-Assisted Laser Desorption/Ionization? Rapid Commun. Mass Spectrom. 1998, 12, 968-974. 